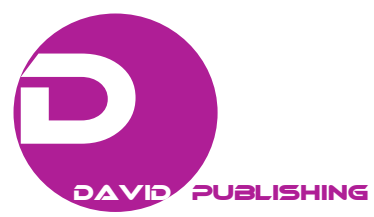

\title{
Linked Micro Parts Referencing System
}

\author{
Philipp Wilhelmi, Christian Schenck and Bernd Kuhfuss \\ Bime (Bremen Institute for Mechanical Engineering), MAPEX Center for Materials and Processing, University of Bremen, Bremen \\ 28359, Germany
}

\begin{abstract}
For simplified processing, micro parts are handled as linked parts. The length of the frame structure of these linked parts can change during a multi-stage production process especially when forming is included. Hence, the positions of individual parts are not constant within the linkage. An individual referencing of the parts becomes necessary in each production step. In this paper, a visual high-speed on-line part referencing system is presented and analyzed. Especially, the trade-off between the highest possible feed velocity and the demand of high accuracy in micro production is taken into account. In addition to this general characterization application related testing with stepwise positioning is performed.
\end{abstract}

Key words: Multi-stage micro part production, linked parts, visual referencing system.

\section{Introduction}

Handling is a challenging task in micro part production and assembly [1], especially with regard to high output rates. To face this challenge multiple micro parts are connected with a frame structure to form linked parts with macro features [2]. The rescaling to macro dimensions helps to overcome micro specific handling problems. Production in multi-stage process chains demands for multiple precise positioning of parts. However, the length of the frame structure can be changed by forming processes like rotary swaging [3]. This results in a shift of the micro parts within the linked part and in relation to the feed system. Consequently, an individual referencing of each single part in every production step becomes necessary. The combination of fragile parts and the requirements of high accuracy and high output rate do not always permit a mechanical referencing solution. Hence, a visual referencing system is developed and investigated. In the presented study the linked parts are based on wire material, see Fig. 1. Preforms are generated within the frame structure by laser material accumulation [4] and formed in a subsequent plunge

Corresponding author: Philipp Wilhelmi, Dipl.-Ing., research fields: handling in micro production, linked parts. rotary swaging process. In order to perform an accurate positioning within the forming tool cavity each preform is referenced individually.

The sensor used in the referencing system is a high-speed line camera. It is triggered based on the position sensor signal of the feed system in a way that lines are acquired at equidistant positions with distance $\Delta \mathrm{x}_{\text {trigger }}$ on the linked part, see Fig. 2. A detailed description is given in Ref. [5]. A perspicuous assumption is that a small trigger distance is favorable for an accurate referencing. However, equidistant triggering entails a dependency between sample rate and feed velocity. In consequence, with higher feed velocities the trigger distance $\Delta x_{\text {trigger }}$ must be increased due to a limited clock rate of the referencing system. By this relationship, the assumption can be derived that the referencing accuracy should be a function of the feed velocity. This assumption is examined by tests with different feed velocities and different trigger distances.

The part referencing system is designed to be combined with different feed systems. These can be for example a feed system with enveloping belts or a gripper based system with one fixed and one reversing gripper, see Fig. 3. The feed systems enable different 


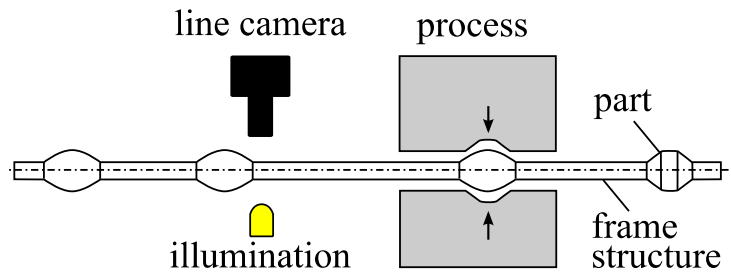

Fig. 1 Processing setup.

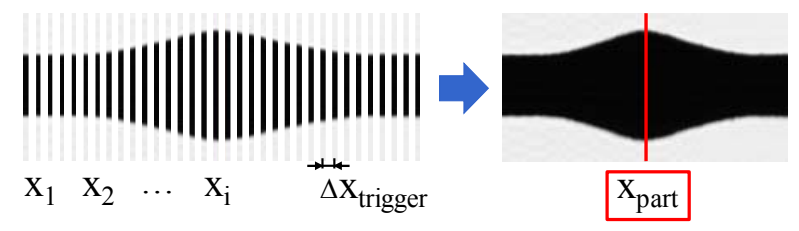

Fig. 2 Basic principle of position triggered data acquisition. [6]
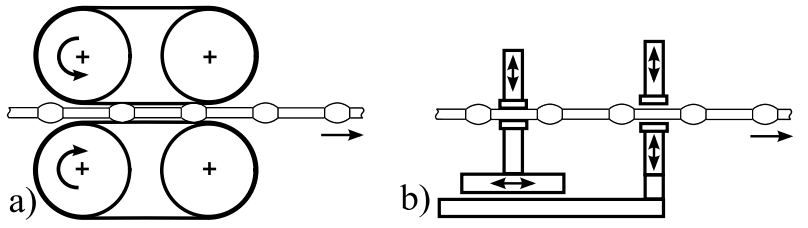

Fig. 3 Linked parts feed system principles. [6]

positioning tasks. The belt-based system is best suited for continuous feeding. The gripper feed system has a limited travel, provides a discontinuous feeding and is optimized for precise positioning.

For the described general characterization of the referencing system, a testing with constant feed velocities is convenient, although in practice other velocity profiles are expected. The linked part should not move in general while processing. Hence, a stepwise positioning, as illustrated in Fig. 4, is applied. An aspired output rate of 400 parts per minute leads to a maximum cycle time of $t_{c y l c e}=0.15 \mathrm{~s}$. If the assumed process time is $t_{\text {process }}=0.05 \mathrm{~s}$, only $t_{\text {positioning }}=0.1 \mathrm{~s}$ remains for positioning. Under the assumption of a constant acceleration, no limitation in velocity and a part distance of $\Delta \mathrm{x}=40 \mathrm{~mm}$ the idealized motion profiles are calculated. For the belt feed system, a maximum velocity of $v_{\max , I}=0.8 \mathrm{~m} / \mathrm{s}$ and a requested acceleration of $a_{1}=16 \mathrm{~m} / \mathrm{s}^{2}$ are determined. The gripper based feed system in contrast makes a reversing motion, while the linked part stands still. Consequently, it must move two times faster and so a velocity of $v_{\max , 2}$ $=1.6 \mathrm{~m} / \mathrm{s}$ and a requested acceleration of $a_{2}=64 \mathrm{~m} / \mathrm{s}^{2}$ are determined.

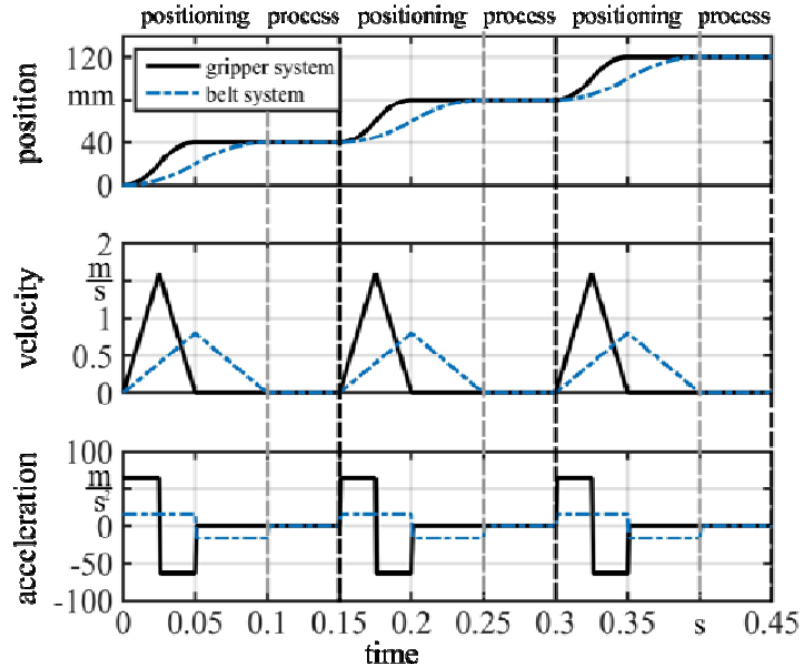

Fig. 4 Positioning profiles.

\section{Referencing System Description}

The system is designed to be integrated in different production set-ups. A trigger module reads out the information from the position sensor of the feed system and generates a position based trigger for the line camera. The part detection module receives the image lines from the camera and detects the part positions within the linked part. These positions can be used subsequently for a positioning of the parts in the tool cavity, see Fig. 5.

The core of the part detection module is an algorithm, which can be executed on standard computers. To keep it as time efficient as possible the image lines are not saved completely, but directly converted to a characteristic value, see Fig. 6. That means instead of buffering 1,024 pixel values of at least 8 bit only a single 64-bit value is stored. In a second step a windowed (window size is $2 \cdot L_{p}$, independently of $\Delta \mathrm{x}_{\text {trigger }}$ ) calculation is applied to the data and the

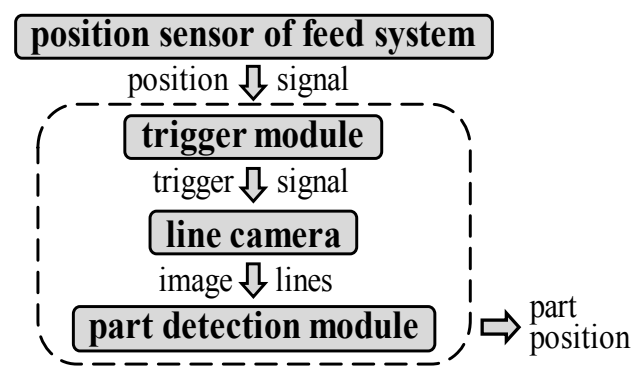

Fig. 5 Modules of micro part referencing system. 
image lines - raw data

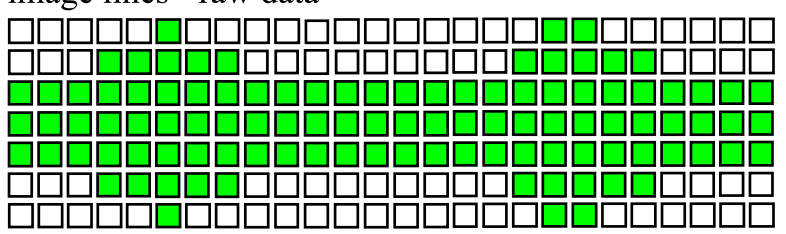

step $1 \downarrow \downarrow \downarrow \cdots$
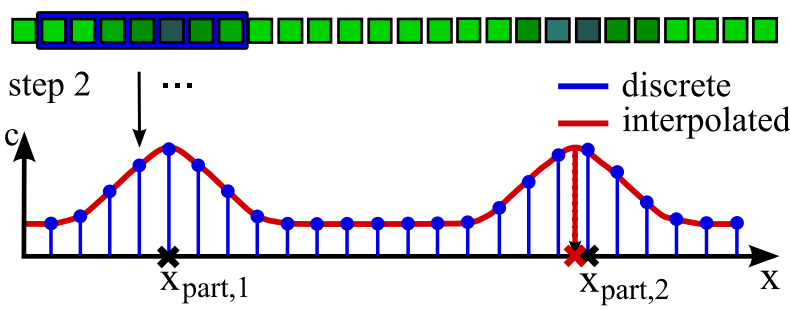

Fig. 6 Scheme of part referencing algorithm. [6]

final characteristic value is derived. The characteristic value in both steps is based on HU-Moments [7]. Finally, a local maximum search is applied to the curve and the locations of the maxima are taken as the part positions. A direct processing of the data delivers the part position with a resolution according to the trigger distance $\Delta \mathrm{x}_{\text {trigger }}$. An additional interpolation that follows the described part referencing increases the resolution. Therefore, each detected maximum value as well as its proceeding and its following sampled values are used to calculate a new maximum by a three-point Gaussian estimator. The final curve in Fig. 6 illustrates the effectiveness of the interpolation. At position $\mathrm{x}_{\mathrm{part}, 1}$ the trigger and the detected part position coincide accidentally. In contrast, at position $x_{\text {part }, 2}$ they do not coincide and the interpolation increases the part referencing accuracy.

\section{Experiments and Discussion}

The tests of the system are performed with a fraction of a line type linked part with seven parts that is fixed in a probe holder, see Fig. 7. The frame structure diameter is $d_{f}=360 \mu \mathrm{m}$, the part diameter is $d_{p}=700 \mu \mathrm{m}$, the part length is about $L_{p} \approx 1,100 \mu \mathrm{m}$ and the overall length is $1=320 \mathrm{~mm}$. During experiments the system is mounted on a high dynamic linear feed axis. The single parts are referenced in motion and with the referenced part positions $x_{\text {part }, i}$ the distances

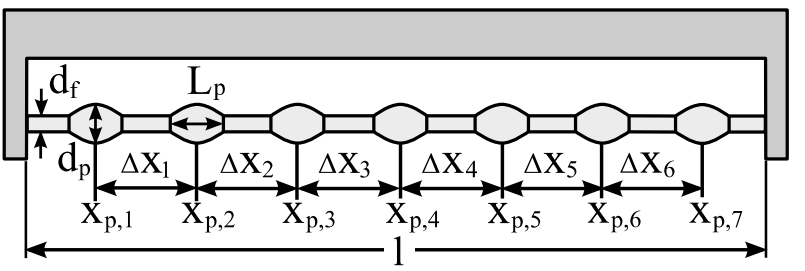

Fig. 7 Linked part for system tests. [6]

$\Delta \mathrm{x}_{i}=\mathrm{x}_{\mathrm{part}, \mathrm{i}+1}-\mathrm{x}_{\mathrm{part}, \mathrm{i}}$ between the parts are calculated. For the evaluation a measurement with the trigger distance $\Delta \mathrm{x}_{\text {trigger }}=1 \mu \mathrm{m}$ is taken as reference. The resulting distances $\Delta \mathrm{x}_{\mathrm{i}, \mathrm{ref}}$ are used to calculate the error $\mathrm{e}_{\Delta \mathrm{xi}}=\Delta x_{i}-\Delta \mathrm{x}_{\mathrm{i}, \mathrm{ref}}$.

Firstly, the influence of the trigger distance on the referencing accuracy is examined at a constant and low feed velocity of $v=0.02 \mathrm{~m} / \mathrm{s}$. To vary the location of every recorded line on the linked part the start point of each measurement within a measurement series is shifted. Figs. 8 and 9 exemplarily show the errors $e_{\triangle x I}$ and $e_{\Delta x 2}$. For every trigger distance $\Delta x_{\text {trigger }}$, the mean value of 10 measurements and the scatter is plotted. With use of the interpolation the mean values of the errors $e_{\Delta x l}$ and $e_{\Delta x 2}$ stay beneath $10 \mu \mathrm{m}$ for a maximum trigger distance of $\Delta x_{\text {trigger }}=400 \mu \mathrm{m}$. This is about

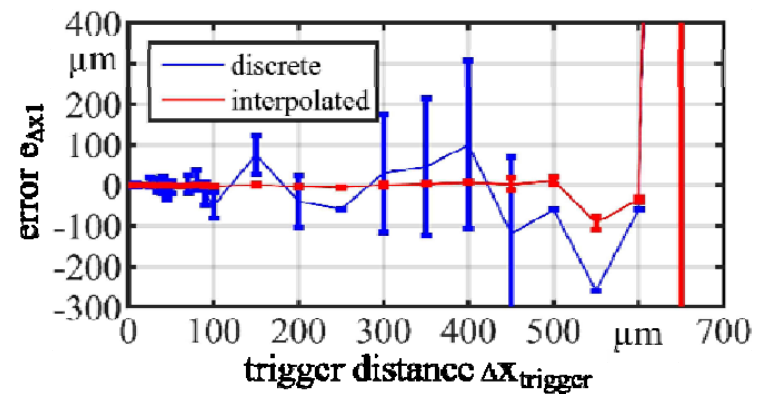

Fig. $8 e_{\Delta x 1}$ measured with different trigger distances, $\Delta \mathrm{x}_{1, \mathrm{ref}}=42,060 \mu \mathrm{m}$. [6]

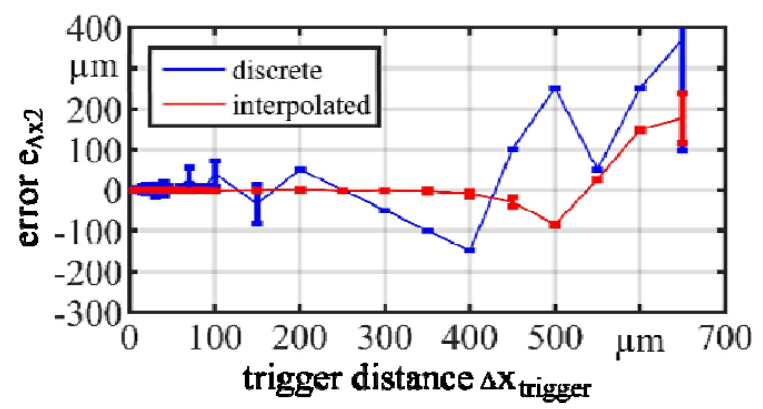

Fig. $9 e_{\Delta \times 2}$ measured with different trigger distances, $\Delta \mathrm{x}_{2}, \mathrm{ref}=41,749 \mu \mathrm{m}$. [6] 


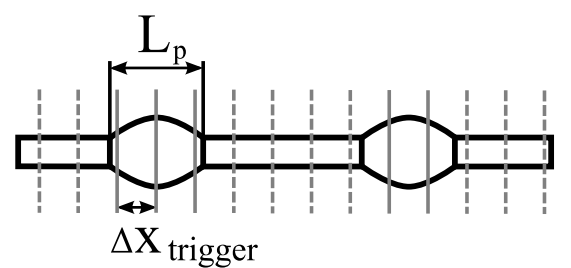

Fig. 10 Minimum trigger distance.
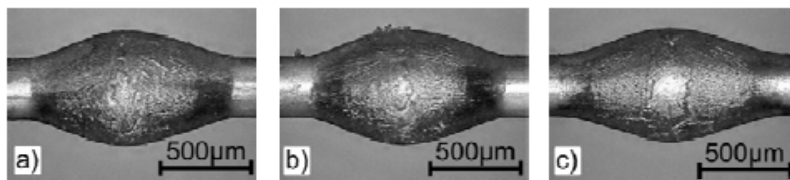

Fig. 11 Images; a) part 1, b) part 2, c) part 3. [6]

half the part length $L_{p} / 2=550 \mu \mathrm{m}$. When considering the effect of the trigger distance on the distribution of the triggered lines on the linked part, as illustrated in Fig. 10, this means that there are always at least two lines on a part. It also has to be taken into account that in the transition zone between wire and part there is only a very small diameter difference, which can explain why the limit is smaller than half the part length.

In contrast to the interpolated referencing, the discrete calculation delivers unsteady values at comparatively low trigger distances. Further, the curve shape varies for the different measured part distances $\Delta \mathrm{x}_{\text {part, } \mathrm{i}}$ which indicates an imprecise measurement without interpolation. This can among others be explained by the varying positions of the image lines on the parts.

If the feed velocity is increased, a given constant trigger distance requires a higher sample rate above a certain level. The highest possible sample rate is limited by the cycle times of the referencing system's modules. These are the clock rate of the trigger generator, the clock rate of the part recognition and the maximum clock rate of the camera which is $f_{\text {camera }}=45$ $\mathrm{kHz}$ in the current case. Based on this clock rate and the maximum expected velocity $v_{\max }=1.6 \mathrm{~m} / \mathrm{s}$ from the described example, a minimum trigger distance of $\Delta \mathrm{x}_{\text {trigger,min }}=v_{\max } / f_{\max }=36 \mu \mathrm{m}$ is possible. For further experiments the trigger distance is set fixed to $\Delta \mathrm{x}_{\text {trigger }}=50 \mu \mathrm{m}$. In Fig. 12 the series of measurements from Fig. 8 are shown in detail up to this value. It can be seen that $\Delta \mathrm{x}_{\text {trigger }}$ has no significant influence on $\mathrm{e}_{\Delta \mathrm{x} 1}$ in case of interpolation in this range.

To illustrate the influence of the velocity exemplary results for the relative errors $\mathrm{e}_{\Delta \mathrm{x} 1}$ and $\mathrm{e}_{\Delta \mathrm{x} 2}$ for the two part distances $\Delta \mathrm{x}_{1}$ and $\Delta \mathrm{x}_{2}$ are illustrated in Figs. 13 and 14 . The mean value stays in a range of $\pm 15 \mu \mathrm{m}$ up to velocity of $v=1.8 \mathrm{~m} / \mathrm{s}$. At the velocity of $v=2 \mathrm{~m} / \mathrm{s}$ the described limit of the clock rate is reached. This is especially indicated by an increasing standard deviation of the interpolated values. As expected the standard deviations with interpolation are smaller than those from the direct evaluation.

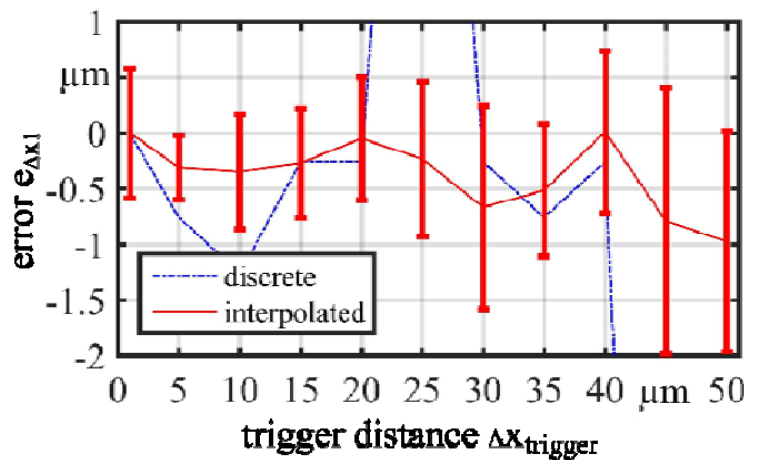

Fig. 12 Detail view of $e_{\Delta x 1}$ measured with different $\Delta x_{\text {trigger }}$. [6]

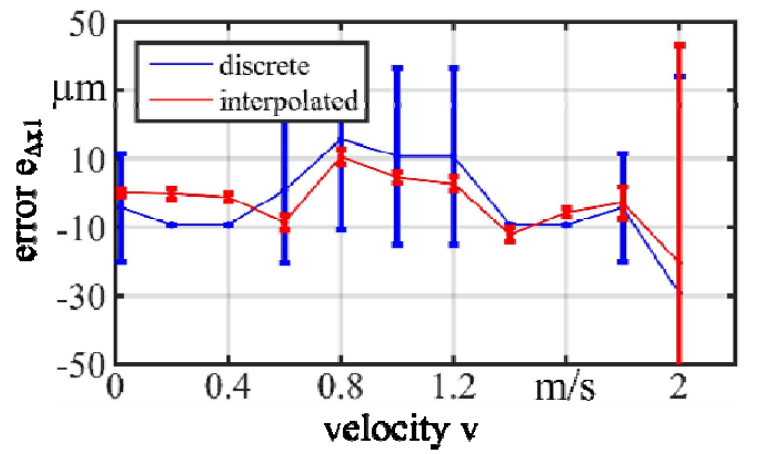

Fig. $13 e_{\Delta x 1}$ at different velocities and $\Delta x_{\text {trigger }}=50 \mu \mathrm{m}$. [6]

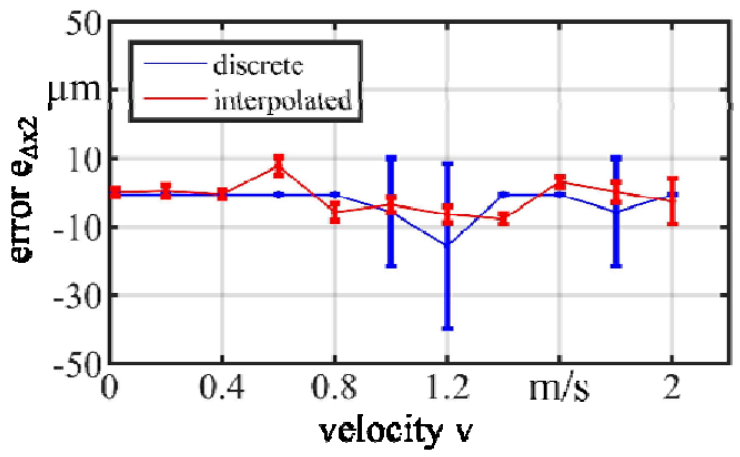

Fig. $14 e_{\Delta x 2}$ at different velocities and $\Delta x_{\text {trigger }}=50 \mu \mathrm{m}$. [6] 
Another finding that can be derived from the study is that up to a velocity of $v=0.4 \mathrm{~m} / \mathrm{s}$ the errors $\mathrm{e}_{\Delta \mathrm{x} 1}$ and $\mathrm{e}_{\Delta \times 2}$ are even lower and the mean error is below $1.5 \mu \mathrm{m}$. At higher velocities the systematic error is slightly higher and differs for the different part distances $\Delta \mathrm{x}_{\mathrm{i}}$. This is observed not only for the two illustrated part distances but also with all six measured distances. At this point, it cannot be determined whether this effect is caused by the referencing system or if it is caused by the test set up or the probe itself. For higher velocities, the appearance of dynamic effects cannot be precluded. Hence, an influence of the acceleration force on the probe and the probe holder is possible. Regardless of the systematic error, the standard deviation stays on a low level until the clock rate limit is reached. This is illustrated in Fig. 15, where the standard deviations of all the six measured distances are averaged in one diagram.

After this general characterization more practice related tests are performed and only the results with interpolation are further considered. For these tests, the same probe and probe holder is used but no longer moved with continuous velocity, but with a stepwise velocity profile as described in the introduction. The average part distance, which is about $\Delta \mathrm{x} \approx 40 \mathrm{~mm}$, is used for the distance of one step. In the start position the distance between the first part and the camera position is half the part distance $\Delta x / 2 \approx 20 \mathrm{~mm}$. From this starting position seven steps are performed to measure $x_{1}$ to $x_{6}$. The described triangular velocity profile is used, so that the highest velocity should be reached, when a part passes the camera. The measurements are performed for four constant acceleration settings from $a_{1}=16 \mathrm{~m} / \mathrm{s}^{2}$ to $a_{4}=64 \mathrm{~m} / \mathrm{s}^{2}$. Ten repeated measurements are performed for each parameter set. For the evaluation again the errors $\mathrm{e}_{\Delta \mathrm{x} 1}$ and $\mathrm{e}_{\Delta \times 2}$ are determined, see Figs. 16 and 17. The mean values of $e_{\Delta x 1}$ and $e_{\Delta \times 2}$ are in the same range as for the tests with constant velocity, but again no clear tendency can be observed.

To get a better impression the standard deviations of all the six measured part distances is averaged in one

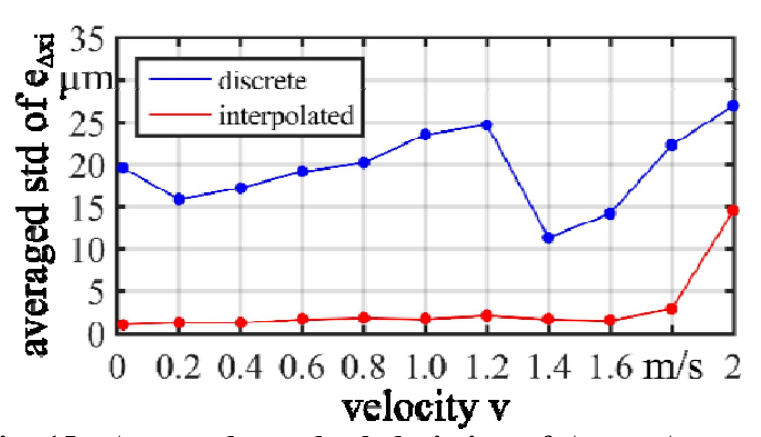

Fig. 15 Averaged standard deviation of $\Delta x_{1}$ to $\Delta x_{6}$ as a function of feed velocity. [6]

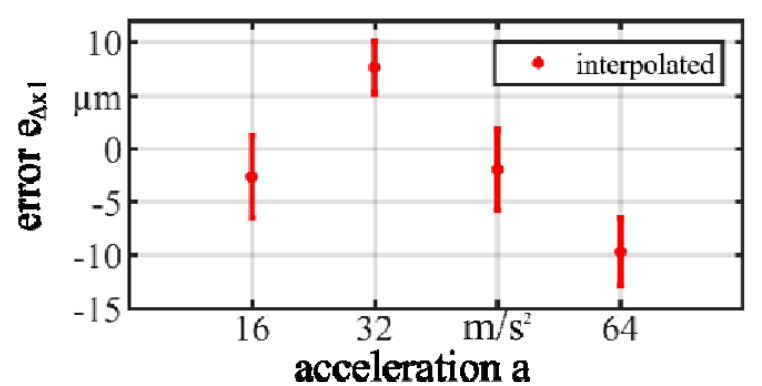

Fig. $16 e_{\Delta x 1}$ at different accelerations and $\Delta x_{\text {trigger }}=50 \mu \mathrm{m}$.

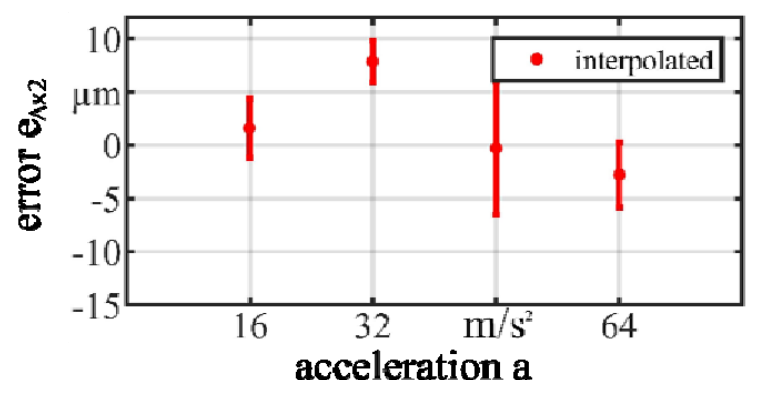

Fig. $17 e_{\Delta \times 2}$ at different accelerations and $\Delta x_{\text {trigger }}=\mathbf{5 0} \mu \mathrm{m}$.

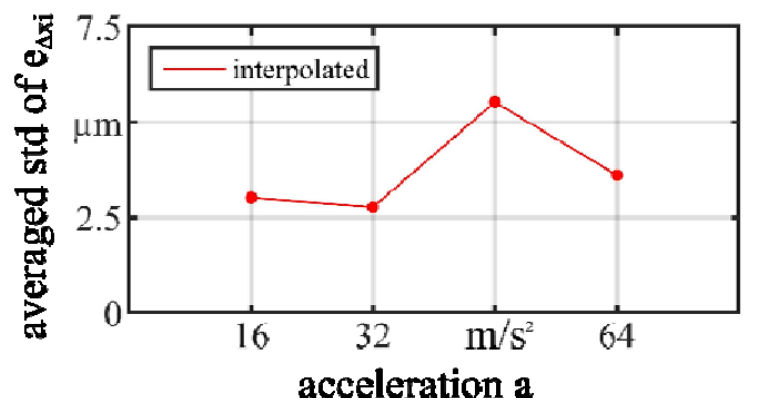

Fig. 18 Averaged standard deviation in dependency of acceleration.

diagram, see Fig. 18. In all cases it is below $6 \mu \mathrm{m}$, but in comparison to the tests with constant velocity the values are varying more. Nevertheless, there is no clear trend. That underlines the assumption made before, that dynamic effects may influence the results and it is still unclear whether the observed variations are caused 
by the probe and probe holder or by the referencing system itself.

\section{Conclusions}

A new developed referencing system for linked micro parts was investigated for appearing errors with regard to high velocities. Besides the direct position calculation, an interpolation based on HU-Moments was tested. The following results were obtained:

(1) Increasing the trigger distance is an appropriate measure to raise the velocity limit of the visual part referencing system, when using the developed interpolation method and respecting the described boundary conditions. For the presented case, it goes along with an acceptable loss in accuracy;

(2) The developed referencing system is able to reference the micro parts within a linked part with high accuracy even at high velocities until the clock rate limit is reached. Considering the mean values, it can be stated that up to a velocity of $\mathrm{v}=0.4 \mathrm{~m} / \mathrm{s}$ the error of detected part positions is even smaller. Here further experiments are necessary to determine the exact reason and whether it is related to the referencing system or the test set up. Considering the velocity range from above $\mathrm{v}=0.4 \mathrm{~m} / \mathrm{s}$ up to the limit of $\mathrm{v}_{\max }=1.6 \mathrm{~m} / \mathrm{s}$ given by the limited clock rate, a loss of accuracy with increasing velocity cannot be observed;

(3) The additional more practice related tests confirm the results from the general characterization. For these tests, a positioning with triangular velocity profiles and consequently constant acceleration is applied. The acceleration values are chosen in order to reach maximum velocities similar to the general characterization. For this stepwise positioning there is only a very small loss in accuracy independent of the applied accelerations;

\section{Acknowledgements}

(1) The authors gratefully acknowledge the financial support by Deutsche Forschungsgemeinschaft (DFG, German Research Foundation) for Subproject C5 "Linked Parts" within the SFB 747 (Collaborative Research Center) "Micro Cold Forming-Processes, Characterization, Optimization". Further we thank Subproject A3 "Material Accumulation" for the production of the linked parts containing the preforms.

(2) This paper is a revised and extended version of reference [6].

\section{References}

[1] Van Brussel, H., Peirs, J., Reynaerts, D., Delchambre, A., Reinhart, G., Roth, N., Weck, M., and Zussman, E. 2000. "Assembly of Microsystems." CIRP AnnalsManufacturing Technology 49 (2): 451-72.

[2] Kuhfuss, B., Moumi, E., Tracht, K., Weikert, F., Vollertsen, F., and Stephen, A. 2011. "Process Chains in Microforming Technology Using Scaling Effect." In Proceedings of the 14th International ESAFORM Conference on Material Forming, 535-40.

[3] Wilhelmi, P., Moumi, E., Foremny, E., Kuhfuss, B., and Schenck, C. 2013. "Feeding and Positioning of Linked Parts in Micro Production Chains." In Proceedings of International Conference on Advanced Manufacturing Engineering and Technologies, 75-84.

[4] Bruenning, H. 2013. "Thermal Free Form Heading." In Micro Metal Forming, edited by Vollertsen, F., et al. Springer, 188-99.

[5] Foremny, E., Bloem, A., Wilhelmi, P., Schenck, C., and Kuhfuss, B. 2014. "Vision Based Feeding System for Linked Parts." 9th International Conference on Micromanufacturing, No. 9.

[6] Wilhelmi, P., Foremny, E., Bloem, A., Schenck, C. and Kuhfuss, B. 2016. "High Speed Referencing System for Linked Micro Parts." In Proceedings of the 11th International Conference on Micro Manufacturing, Paper\# 19.

[7] Hu, M. 1962. "Visual Pattern Recognition by Moment Invariants." IRE Transaction on Information Theory 8 (2): 179-87. 